\title{
Cryptogenic Organizing Pneumonia
}

National Cancer Institute

\section{Source}

National Cancer Institute. Cryptogenic Organizing Pneumonia. NCI Thesaurus. Code C62586.

A form of organizing pneumonia with a non-infectious etiology. Excessive proliferation of granulation tissue occurs in alveolar ducts and alveoli and primarily causes injury to alveolar walls, but bronchioles may be affected. 